\section{CIÊNCIA CIDADÃ EM TEMPOS DE EMERGÊNCIAS: INICIATIVAS BRASILEIRAS ANTE A PANDEMIA DA COVID-19}

\author{
Sarita Albagli \\ Instituto Brasileiro de Informação em Ciência e Tecnologia - IBICT \\ http://orcid.org/0000-0003-0030-8964 \\ sarita.albagli@gmail.com \\ Luana Rocha \\ Instituto Brasileiro de Informação em Ciência e Tecnologia - IBICT \\ http://orcid.org/0000-0001-5602-0375 \\ luaroc@gmail.com
}

Cómo citar este artículo/Citation: Albagli, Sarita; Rocha, Luana (2021). Ciência cidadã em tempos de emergências: iniciativas brasileiras ante a pandemia da COVID-19. Arbor, 197(799): a589. https://doi.org/10.3989/arbor.2021.799004

Recibido: 20 noviembre 2020. Aceptado: 22 febrero 2021. Publicado: 9 abril 2021

RESUMO: A pandemia da COVID-19 evidencia possibilidades, limites e novos desafios à abertura da ciência. Neste artigo, apresentam-se resultados de pesquisa que objetivou contribuir para o entendimento do papel da ciência cidadã, uma das vertentes da ciência aberta, para lidar com as questões postas pela pandemia. Além de revisão da literatura, o estudo envolveu a identificação e a categorização de iniciativas cidadãs dessa natureza, no Brasil, durante o período de eclosão da COVID-19. Observa-se o aprendizado mútuo entre as abordagens da ciência cidadã e as ações participativas para redução de riscos de desastre. De um lado, fortalece o papel da produção cidadã de dados, informações e conhecimentos relevantes à prevenção, resiliência e gestão desses riscos; e, de outro, alarga o escopo da ciência cidadã para o enfrentamento de situações de emergência e desastre, ao mesmo tempo em que reforça o caráter situado dessa abordagem.

PALAVRAS-CHAVE: ciência cidadã, COVID-19, redução de riscos de desastres.

\section{CIENCIA CIUDADANA EN TIEMPOS DE EMERGENCIA: INICIATIVAS BRASILEÑAS ANTE LA PANDEMIA DE LA COVID-19}

\author{
CITIZEN SCIENCE DURING \\ EMERGENCIES: BRAZILIAN \\ INITIATIVES TO FACE THE \\ COVID-19 PANDEMIC
}

Copyright: (C) 2021 CSIC. Este es un artículo de acceso abierto distribuido bajo los términos de la licencia de uso y distribución Creative Commons Reconocimiento 4.0 Internacional (CC BY 4.0).

RESUMEN: La pandemia de la COVID-19 evidencia posibilidades, límites y nuevos desafíos para la ciencia abierta. En este artículo se presentan los resultados de una investigación que tuvo como objetivo contribuir a la comprensión del papel de la ciencia ciudadana, una de las vertientes de la ciencia abierta, en el abordaje de los problemas plantados por la pandemia. Para ello, se llevó a cabo una revisión de literatura especializada y se acometió la identificación y la categorización de estas iniciativas ciudadanas en Brasil durante el periodo del brote de COVID-19. Los resultados de esta investigación muestran un aprendizaje mutuo entre los abordajes de la ciencia ciudadana y las acciones participativas para reducir los riesgos de desastres. Este aprendizaje, por un lado, fortalece el papel de la producción ciudadana de datos, información y conocimiento relevante para la prevención, resiliencia y gestión de estos riesgos; $y$, por otro lado, dilata el alcance de la ciencia ciudadana para afrontar situaciones de emergencia y desastre, al tiempo que refuerza el carácter situado de este enfoque.

PALABRAS CLAVE: ciencia ciudadana, COVID-19, reducción del riesgo de desastres. 
ABSTRACT: The COVID-19 pandemic highlights the possibilities, limits, and new challenges for Open Science. This article presents the results of research that sought to contribute to the understanding of the role of citizen science, one of the axes of Open Science, to deal with issues posed by the pandemic. In addition to a literature review, the study involved identification and categorization of these citizen initiatives in Brazil during the COVID-19 outbreak. Mutual learning can be seen between citizen science approaches and participatory actions to reduce risks

\section{INTRODUÇÃO}

A eclosão da pandemia da COVID-19, em 2020, tornou ainda mais evidente e dramático o agravamento da crise socioambiental global e suas múltiplas repercussões, bem como seu caráter antropogênico e sistêmico. Dela derivou uma crise sanitária e humanitária sem precedentes, acometendo milhões de pessoas em todo o mundo ${ }^{1}$.

As pandemias podem ser consideradas situações de emergência e desastre, ante a magnitude, o caráter atípico e a dificuldade de respostas isoladas, devido à sua propagação transfronteiras, com repercussões além daquelas que se enquadram como emergências de saúde pública de abrangência internacional.

A pandemia da COVID-19 pode ser definida como um «desastre biológico com elementos tecnológicos e societais atuantes em sua propagação» (Rodrigues et al., 2020). Trata-se mais propriamente de uma sindemia, envolvendo aspectos e consequências ambientais, sociais, culturais, econômicos e técnicos, que se retroalimentam (Horton, 2020). Projeta-se aí a noção de one health (Black e Butler, 2014), denotando a ideia de que há um desequilíbrio que afeta a saúde de tudo e todos que integram a coexistência planetária ecossistemas, seres vivos e inanimados, sistemas econômicos e sociais, bem como países, regiões, grupos sociais e indivíduos -, colocando a centralidade da proteção da vida em sua diversidade, bem como dos valores de (con)vivência que a sustentam.

Hoje se impõe, portanto, a redefinição dos conceitos de emergência e desastre socioambientais e sanitários. De eventos pontuais e localizados, passam a caracterizar-se como processos que se conectam em escala planetária, em suas causas e efeitos. Já não se encerram em si mesmos, desdobrando-se em situações de crise sucessivas, ainda que com maior inten- from disasters. On the one hand, this learning strengthens the role of citizens' production of data, information, and knowledge relevant for the prevention, resilience to, and management of these risks; and, on the other hand, it broadens the scope of citizen science to confront emergency and disaster situations while reinforcing the situated character of this approach.

KEYWORDS: Citizen science, COVID-19, reduction of risk from disasters.

sidade e perigo em determinados períodos e locais. A ampliação espaço-temporal dessas situações é acompanhada da ampliação de seu escopo e sua escala, tanto em termos quantitativos (frequência e número de afetados), quanto qualitativos (tipos de impactos, riscos e consequências), repercutindo na percepção de risco e vulnerabilidade. Pesam ainda, nesse cenário, os efeitos da globalização com o incremento histórico dos fluxos transnacionais de pessoas, bens e informações.

As estratégias para o enfrentamento dessas situações de emergência e desastre requerem então perspectivas multidimensionais, que permitam capacidade de mitigação e adaptação, resiliência e redução da vulnerabilidade de pessoas, sistemas socioeconômicos e ecológicos. Tais requisitos não se reduzem a respostas em momentos de eclosão e duração desses eventos, devendo estender-se para o estabelecimento de condições para mitigação das causas, bem como para a prevenção de novas situações de crise, de uma perspectiva sistêmica e holística.

Diante desse quadro, assumem caráter estratégico as condições e capacidades para a produção, o acesso, o uso e a análise de dados, informações e conhecimentos qualificados para a efetiva prevenção, monitoramento e intervenção nessas situações. 0 conhecimento científico disponível, ainda que fundamental, não é suficiente para dar conta do desafio, com a extensão e a velocidade necessárias. Do mesmo modo, coloca-se a relevância da sensibilização, o envolvimento e a mobilização de um amplo espectro de atores, em diferentes esferas e escalas, incluindo grupos afetados e vulneráveis, agentes governamentais, sociais e privados.

Ante o pouco conhecimento sobre a doença e seu agente causador, o vírus Sars-CoV-2, as altas taxas de internação hospitalar e de mortalidade, o despreparo

1 Entre o início de fevereiro de 2020 e o final de fevereiro de 2021, isto é, após um ano de pandemia, foram registrados no mundo quase 113 milhões de casos e 2,5 milhões de mortes, de acordo com o levantamento do Centro de Recursos do Coronavírus da Universidade John Hopkins, referência no acompanhamento do avanço da doença no mundo. Os dados são atualizados diariamente e estão disponíveis no portal https://coronavirus.jhu.edu/map.html. 
dos sistemas de saúde nacionais para atender de forma adequada os enfermos e as graves consequências sociais e econômicas que afetam principalmente as populações mais carentes, observa-se o surgimento de variados tipos de iniciativas da sociedade na busca de formas de enfrentamento da crise, de modo complementar e muitas vezes colaborativo com os esforços feitos pela comunidade científica e pelos agentes públicos e privados.

A ciência cidadã - uma das vertentes do movimento pela ciência aberta, cujo foco recai na contribuição e no protagonismo de não cientistas na produção de informações, dados e conhecimentos (Irwin, 1995; Bonney, 2009; Haklay, 2015)-tem potencialmente um papel de destaque nesse cenário. Baseando-se crescentemente em tecnologias digitais, demanda novas infraestruturas de dados e informações, bem como formas e protocolos específicos para sua coleta, organização, disponibilização, acesso e uso.

Este artigo apresenta resultados de pesquisa que objetiva contribuir para o avanço do conhecimento teórico e empírico sobre as possibilidades e limites da ciência cidadã no enfrentamento de situações de emergência sanitária como a da COVID-19. Além de revisão da literatura, o estudo envolveu a identificação e a categorização de iniciativas cidadãs com essa abordagem, no Brasil, para o enfrentamento da pandemia COVID-19 e de suas implicações sociais e econômicas.

\section{CIÊNCIA ABERTA E CIDADÃ ANTE AS NOVAS EMERGÊNCIAS}

A abertura da ciência, em diferentes modalidades -particularmente o acesso aberto às publicações científicas, a agilização do processo de avaliação de artigos submetidos para publicação (fast track), o incentivo aos preprints, a abertura e o compartilhamento de dados de pesquisa- tem sido apontada como crucial para ampliar a circulação de conhecimento, com a rapidez e o volume requeridos ao enfrentamento da pandemia da COVID-19 (Rios, Zheng e Zheng, 2020; Tse, Klug e Todd, 2020).

Cabe refletir, por outro lado, o que a pandemia nos revela sobre as possibilidades, limites e novos desafios à abertura da ciência.

Um primeiro aspecto diz respeito à necessidade de repactuar as relações entre ciência e sociedade. Anteriormente à disseminação da COVID-19, já se observava uma significativa inflexão nessa relação. Assim, se, a partir da Segunda Guerra Mundial, a ciência ganhou status de política pública e merecedora de financiamento do Estado (Velho, 2011), nos tempos mais recentes difundiram-se visões anti-ciência, como os movimentos antivacina, o negacionismo da questão climática e ideários do terraplanismo (Hussain et al., 2018; Abellán-López, 2021).

A pandemia trouxe novos ingredientes a esse cenário. Por um lado, ao tornar o risco e a vulnerabilidade mais visíveis e palpáveis do que a própria emergência climática, reforçou, em alguns setores, o reconhecimento do papel da ciência como parte estratégica no encontro de soluções para a crise. Por outro lado, boa parte da opinião pública em diferentes países manteve-se refratária às evidências científicas e permeável à proliferação das fake science (Oliveira, 2020), acirrando as controvérsias a respeito da gravidade e das estratégias e medidas de enfrentamento da doença. Tornou-se então mais evidente a necessidade de novas linguagens, formas e meios de comunicação, não só em uma via única, da ciência para a sociedade, mas também da escuta da ciência em relação ao que a sociedade tem a dizer e a suas diferentes formas de contribuir (Flores e Asuncion, 2020; Lasser et al., 2020; Metcalfe et al., 2020). Essa questão já fora apontada muito antes da eclosão da pandemia (Irwin, 1995; Lafuente e Estalella, 2015).

Um segundo aspecto, relacionado ao anterior, refere-se ao papel da informação na mudança de comportamento com vistas ao enfrentamento da COVID-19. A informação é uma forma de registro e circulação de conhecimentos que, de outro modo, poderiam ficar encerrados nos indivíduos, nos especialistas, cientistas e laboratórios. Mas a mera disponibilização da informação não move à ação. Com os novos meios e tecnologias digitais de informação e comunicação, que facilitam a circulação ampliada da informação, há uma democratização no acesso. Mas é preciso também democratizar as formas e as competências para se apropriar e dar uso a esses dados e informações, assim como para filtrar e julgar sua procedência e veracidade, a chamada competência crítica em informação (Bezerra et al., 2019)

É também necessário mobilizar as dimensões subjetiva e ético-valorativa, de modo a que a informação contribua para promover a responsabilidade social, a empatia, a solidariedade e o respeito à diversidade.

Nesse contexto, a ciência cidadã pode desempenhar um papel significativo. 


\subsection{A ciência cidadã e a redução de riscos de desastres}

Abordagens de ciência cidadã vêm contribuindo para incorporar a participação voluntária e a contribuição de comunidades afetadas na provisão e análise de grandes quantidades de dados heterogêneos, tornando mais robustos os conhecimentos daí derivados. A conjugação entre diferentes experiências, formações e habilidades, bem como os processos de aprendizado social daí decorrentes, é relevante na construção de uma inteligência coletiva e seu uso em diferentes tipos de inovação (Dickinson et al., 2012).

Critica-se, por outro lado, o fato de que iniciativas de ciência cidadã "têm normalmente ocorrido em locais afluentes, excluindo as populações não alfabetizadas ou letradas e que vivem em áreas remotas» (Comandulli et al., 2016: 36). Estudos revelam que a maior parte dos participantes de iniciativas de ciência cidadã, em seus moldes mais tradicionais, é composta por homens brancos, bem situados economicamente, com acesso à internet e aparelhos de celular, e concentrados em países de economia avançada do Norte global (Muklay, 2015). Advoga-se alternativamente pelo conceito de ciência cidadã «extrema» (Stevens et al., 2014) ${ }^{2}$, cujo foco é «permitir que qualquer comunidade, em qualquer lugar do mundo [...] comece um projeto de ciência cidadã para lidar com suas próprias questões.» (Comandulli et al., 2016: 36).

Nesse sentido, para além das motivações que balizam iniciativas de voluntariado em geral -diversão, prazer, altruísmo, interação social-, o engajamento de não especialistas em iniciativas de ciência cidadã é movido também pelo desejo de intervir mais ativamente em questões que interferem diretamente em suas vidas, cuja decisão se baseia frequentemente e de modo restrito à opinião de especialistas, ampliando o engajamento e a repercussão social dessas ações.

Iniciativas e métodos de ciência cidadã vêm sendo crescentemente mobilizados para o enfrentamento e a redução de riscos de desastres (RRD) e situações de emergência ${ }^{3}$, contribuindo para o mapeamento e a prevenção de riscos, bem como para o desenvolvimento de estratégias e sistemas de alerta, monitoramento e gestão de impactos. Apontam-se como possíveis contribuições da ciência cidadã nesses casos: fonte de dados e informações relevantes ao planejamento e à realização de ações; ferramenta de comu- nicação, facilitando e mobilizando atuações conjuntas e, logo, fortalecendo o capital social; recurso para comunidades afetadas visibilizarem seus conhecimentos, aprendizados e pontos de vista, frequentemente desafiando narrativas dominantes (Marchezini et al., 2017; Hicks et al., 2019; Calyx, 2020).

A combinação desses dois conjuntos heterogêneos de abordagens -ciência cidadã e redução de riscos de desastres- tem propiciado aprendizados recíprocos e benefícios mútuos. Por um lado, a ciência cidadã contribui para conferir maior robustez à produção de dados e informações pertinentes à prevenção e gestão de riscos, cuja utilidade e relevância não se restringem aos momentos críticos e temporalmente limitados de resposta a esses eventos. Por outro lado, a ciência cidadã tem seu escopo de intervenção e seu campo semântico alargados para além da esfera estrita da ciência, considerando a produção de conhecimentos em sentido amplo, segundo o contexto e os objetivos para os quais é mobilizada, daí resultando "um tipo diferente de ciência e um tipo diferente de conhecimento» (Strasser et al., 2018 apud Hicks et al., 2019: 4). Dessa perspectiva, a ciência cidadã «coloca cidadãos no centro de um processo que gera novo conhecimento para a redução dos riscos de desastre» (Hicks et al., 2019:4).

O ponto de interseção e complementaridade entre ambas as abordagens é a participação cidadã, seja por motivações e esforços altruístas de caráter voluntário e humanitário, seja como forma de intervenção, visibilização e resiliência de grupos afetados e vulneráveis nessas situações de emergência. Essa combinação é reforçada pelo Marco de Sendai para a Redução do Risco de Desastres 2015-2030 (Sendai Framework for Disaster Risk Reduction), estabelecido no âmbito das Nações Unidas, que tem dentre seus princípios básicos: (1) a necessidade de haver uma compreensão -logo, conhecimento- sobre esses riscos, visando apoiar a prevenção, preparação, resposta e recuperação de seus impactos; e (2) a centralidade de uma abordagem inclusiva, participativa e propícia à contribuição voluntária que promova o engajamento cidadão nessas ações (Rodrigues et al., 2020).

No campo da RRD, destacam-se as abordagens que dão centralidade às pessoas e grupos atingidos ('people-centered' $D R R$ ), em que comunidades afetadas são

2 Esse é o enfoque do grupo de pesquisa interdisciplinar excites, criado em 2011, na University College London.

3 «Tanto a OMS quanto a PNPDEC utilizam cinco fases para definir o ciclo do desastre: prevenção, mitigação, preparação, resposta e recuperação [...].» (Rodrigues et al., 2020) 
ativas no mapeamento de riscos, contribuindo para o aumento da resiliência e a tomada de decisão em âmbitos individual, comunitário e das políticas públicas. Avalia-se como virtuosa, nesses contextos, a colaboração entre as informações e conhecimento situados e baseados na experiência aportados por comunidades afetadas e sob risco e os conhecimentos derivados da pesquisa científica, o que também contribui para democratizar a produção e o acesso ao conhecimento científico (Hicks et al., 2019; Albagli e Iwama, no prelo). Isso é condizente, embora não suficiente, com a perspectiva de uma ciência cidadã «extrema».

No caso da COVID-19, esses aportes são ainda mais necessários, considerando o amplo desconhecimento ainda existente sobre a doença e a suas implicações sociais, políticas, econômicas e éticas, bem como a insuficiente resposta por parte dos poderes públicos, como é o caso do Brasil.

Em diversos países observa-se a disseminação de iniciativas de ciência cidadã para a provisão e o registro informações, de modo participativo, a partir de experiências diferenciadas de reação e enfrentamento da doença, incluindo desde dados sobre sintomas e modos de recuperação até sobre a distribuição territorial de incidências, oferta de serviços de apoio e redes de solidariedade, entre outros (Clinio et al., 2020; Bowser, Parker e Long, 2020). Essas iniciativas recorrem frequentemente a aplicativos de celular e programas de geolocalização, a exemplo de outras frentes de ciência cidadã, adotando plataformas pré-existentes ou desenvolvidas especialmente com essa finalidade.

Um exemplo é a iniciativa australiana BeatCovid19Now ${ }^{4}$, que faz uso de um aplicativo para celular batizado de Symptom Tracker para rastrear casos da doença com a colaboração da população - que contribui de maneira anônima sobre seu estado de saúde. 0 site foi desenvolvido por uma equipe multidisciplinar formada por agentes de saúde pública, cientistas, especialistas em tecnologia da informação (TI) e pessoal da Swinburne University of Technology, em parceria com a empresa de serviços tecnológicos ARQ Group, o COVID-19 Hackathon e profissionais de marketing.
Iniciativas com abordagens similares se disseminaram amplamente, como o CoronAPP (Dinamarca), Corona Israel ${ }^{6}$, Corona Report ${ }^{7}$ (Escócia) e o COVID-19 Citizen Science $^{8}$ (Estados Unidos).

Destaca-se também o papel das inovações sociais no enfrentamento da pandemia, incluindo formas inovadoras de redes de apoio e solidariedade até iniciativas de produção de equipamentos de proteção individual (EPI) e de cuidados (como respiradores) de baixo custo e com hardware aberto. Estas últimas conectam frequentemente instituições de ensino e pesquisa e as chamadas comunidades makers, que se desenvolvem fora dos espaços formais e reconhecidos de produção científicas, logrando envolver e mobilizar a contribuição daqueles que não têm assento ou não encontram oportunidades nos espaços tradicionais (Martins, 2017; Smith et al., 2017; Martins e Albagli, 2019).

Neste grupo estão o coletivo internacional de código aberto HelpfulEngineers ${ }^{9}$, que reúne diversos projetos de tecnologia aberta voltados para o combate à pandemia, a Coronavirusmakers ${ }^{10}$, uma rede de integrantes do movimento maker e especialistas em tecnologia aberta que busca desenvolver e fabricar equipamentos como ventiladores e EPIs para suprir a demanda dos sistemas de saúde, e a OpenCovid19 Initiative ${ }^{11}$, projeto do Just One Giant Lab (JOGL) para desenvolver ferramentas e metodologias abertas e de baixo custo para o enfrentamento da pandemia, do qual fazem parte mais de 4 mil pessoas voluntárias.

\section{INICIATIVAS NO BRASIL}

Apresentam-se aqui os procedimentos adotados e os resultados obtidos no levantamento e caracterização de iniciativas de ciência cidadã e afins para o enfrentamento da COVID-19, no ano de 2020.

\subsection{Metodologia}

Conforme já assinalado, neste estudo, adotou-se um conceito mais alargado de ciência cidadã, compreendendo tanto iniciativas autodenominadas como tal, como experiências cujo foco recai na mobilização da contribuição voluntária e participativa de não

\footnotetext{
4 https://beatcovid19now.org

5 https://www.coronapp.dk

6 https://coronaisrael.org/en/

7 https://www.coronareport.global/

8 https://Covid19.eurekaplatform.org/

9 https://helpfulengineering.org/

10 https://www.coronavirusmakers.org/

11 https://app.jogl.io/program/openCovid19
} 
cientistas ou especialistas na produção e no compartilhamento de dados e informações relevantes ao enfrentamento da COVID-19 e suas implicações sociais e econômicas. Não foram consideradas iniciativas circunscritas aos ambientes acadêmico e governamental que não envolvessem a participação e a colaboração ativa desses públicos mais amplos, como portais de instituições de pesquisa e órgãos governamentais, ou mesmo redes restritas a pesquisadores.

O intuito foi identificar e caracterizar a diversidade de experiências e tipos de abordagens de iniciativas cidadãs, ou com a participação cidadã, de produção de dados e informações sobre a pandemia no país, que se utilizaram de plataformas online, sem a pretensão de fazer um levantamento extensivo de todas as ações em curso.

A coleta dos dados sobre as iniciativas foi realizada no período de 25 de março a 3 de novembro de 2020, a partir de: buscas na Internet; notícias divulgadas por meio eletrônico, como portais jornalísticos; posts em redes sociais e grupos de Facebook criados para a mobilização de pessoas interessadas em contribuir com iniciativas de enfrentamento da pandemia; e plataformas que reúnem informações sobre iniciativas de ciência cidadã e ciência aberta, algumas das quais criadas com foco específico na COVID-19. Para as buscas, utilizou-se a combinação de diferentes palavras-chave relativas ao tema (COVID-19, ciência cidadã, iniciativa cidadã, respiradores, equipamentos de proteção individual, EPIs, entre outras).

A partir desse levantamento, as iniciativas que atenderam aos critérios de seleção estabelecidos foram agrupadas, conforme similaridade de atuação, em três categorias de atuação e análise, definidas pela pesquisa, quais sejam:

a. Dados e informações - Projetos, iniciativas e plataformas de coleta, monitoramento, disponibilização e geovisualização (mapas) de dados sobre a doença, incluindo os que se autodenominam como ciência cidadã, desenhados de forma a facilitar a produção participativa e a apropriação social da informação, mobilizando a contribuição de indivíduos e grupos sociais mais afetados e vulneráveis.

b. Fabricação cidadã - Desenvolvimento colaborativo de protótipos e fabricação de equipamentos com tecnologias abertas e de baixo custo, relevantes ao enfrentamento da pandemia, tais como respiradores, ventiladores, equipamentos de proteção individual (EPIs), entre outros, para doação a profissionais e instituições de saúde e utilização pela sociedade em geral. A fabricação geralmente está associada ao movimento maker e redes de inovação cidadã.

c. Redes de solidariedade e apoio assistencial Redes online, muitas das quais com atuações locais e de vizinhança solidária, para registro e geovisualização de dados, de modo colaborativo, visando a visibilização e troca de informações sobre demanda e oferta de apoio assistencial, médico e psicológico voluntário, sobretudo para populações mais vulneráveis ao vírus ou em situação de vulnerabilidade social e econômica. $\mathrm{O}$ foco aqui são ações que buscam conectar diferentes atores através da informação, construindo conhecimento para ação, para que pessoas possam buscar e prover apoio.

Cabe ressaltar que as fronteiras entre essas categorias não são estanques, e uma mesma iniciativa pode apresentar características que a enquadrariam em mais de um grupo. No entanto, para fins de análise, optou-se por alocar cada projeto em uma única categoria, de acordo com seu objetivo principal. Ressalte-se ainda que, nas três categorias, algumas iniciativas tiveram como objetivo realizar e disponibilizar um mapeamento de iniciativas afins.

Foi também analisado se as iniciativas possuíam ou não vínculos institucionais formais, isto é, se eram mobilizadas por ou tinham apoio oficial de instituições - como universidades, centros de pesquisa, organizações não governamentais (ONGs) - ou se eram independentes, resultando da mobilização autônoma de indivíduos ou coletivos já existentes ou formados especificamente para o enfrentamento da crise.

\subsection{Resultados e discussão}

No total, foram analisadas 71 iniciativas, distribuídas segundo as três categorias adotadas na pesquisa $(\text { Tabela } 1)^{12}$. A categoria com maior número de iniciativas foi a de redes de solidariedade e apoio (36), seguida de fabricação cidadã (19) e dados e informação (15). Há ainda uma iniciativa não incluída nas tabelas, que é analisada à parte, mais adiante, por não se enquadrar em nenhuma das três categorias.

12 Conjunto de dados disponibilizado, conforme registro indicado ao final do artigo. 
Tabela 1. Iniciativas conforme categorias ${ }^{13}$

\begin{tabular}{|l|c|}
\hline \multicolumn{1}{|c|}{ Foco } & Total \\
\hline Dados e informações & 15 \\
\hline Fabricação cidadã & 19 \\
\hline Redes de solidariedade e apoio & 36 \\
\hline Total & 70 \\
\hline
\end{tabular}

Fonte: Elaboração própria

As experiências e abordagens utilizadas pelas iniciativas em cada categoria são bastante diversas entre si, como será discutido a seguir. Conforme já assinalado, em comum a todas elas, está o uso de plataformas digitais para produção e compartilhamento de dados, informações e conhecimentos, boa parte das quais associadas à formação de redes de colaboração e voluntariado.

Com relação ao vínculo institucional (Tabela 2), observa-se um alto número de iniciativas independentes, principalmente nas categorias de redes de solidariedade e apoio (em que 22 das 36 iniciativas analisadas não têm vínculo institucional) e de fabricação (mais da metade não tem vínculo institucional). Entre as instituições às quais as iniciativas analisadas estão vinculadas, têm-se ONGs, universidades (em alguns casos, laboratórios ou grupos de pesquisa específicos), instituições de pesquisa científica, startups, fablabs, espaços makers e órgãos públicos da administração direta.

Tabela 2. Iniciativas conforme vínculo institucional ${ }^{14}$

\begin{tabular}{|l|c|c|c|c|}
\hline \multicolumn{1}{|c|}{ Foco } & $\begin{array}{c}\text { Com } \\
\text { vínculo }\end{array}$ & Independentes & $\begin{array}{c}\text { Não identi- } \\
\text { ficado }\end{array}$ & Total \\
\hline $\begin{array}{l}\text { Dadose } \\
\text { informa- } \\
\text { ções }\end{array}$ & 6 & 8 & 1 & 15 \\
\hline $\begin{array}{l}\text { Fabricação } \\
\text { cidadã }\end{array}$ & 8 & 11 & 0 & 36 \\
\hline $\begin{array}{l}\text { Redes de } \\
\text { solidarie- } \\
\text { dade e } \\
\text { apoio }\end{array}$ & 12 & 24 & 19 & 70 \\
\hline Total & 26 & 43 & & \\
\hline
\end{tabular}

Fonte: Elaboração própria.

Verificou-se também a formação de redes com diferentes escalas de atuação -local, interestadual e mes- mo internacional-, conectando e formando vínculos colaborativos entre diferentes iniciativas.

Observou-se, por sua vez, uma pequena proporção de iniciativas nos moldes tradicionais da ciência cidadã, o que é congruente com o verificado em pesquisa anterior que detectou a experiência ainda tímida no Brasil nesse campo, em termos tanto numérico como de escopo temático (Albagli e Rocha, 2021). Essa mesma pesquisa mostrou que iniciativas e projetos de pesquisa em ciência cidadã desenvolvidos no Brasil têm validou-se sobretudo da colaboração cidadã no levantamento e no registro de dados em apoio à pesquisa científica, não necessariamente recorrendo a metodologias de cocriação, o que também ficou evidenciado neste estudo.

A seguir, em cada categoria, algumas iniciativas são descritas detalhadamente, a título de exemplificação.

\subsubsection{Dados e informações}

Em dados e informação, estão iniciativas cidadãs ou com participação cidadã, de coleta, divulgação e visualização de dados sobre o avanço da pandemia, tais como indicadores de saúde (incluindo capacidade de atendimento dos serviços de saúde) e monitoramento do número de casos e de óbitos.

Aqui estão incluídos sites com levantamento e tratamento de dados, além de visualização dos números de caso e de mortes por localidade, bem como a disponibilização de modelagens e APIs (interface de programação de aplicação, em português) abertas que possibilitem o monitoramento do avanço da pandemia. Há também iniciativas de produção de análises e de crowdsourcing (produção coletiva de dados) para vigilância epidemiológica. Várias dessas iniciativas estão ligadas a instituições de ensino e pesquisa (universidades, em especial), mas a maioria não possui vínculo institucional.

O projeto Especial Covid-19 da plataforma Brasil.lo ${ }^{15}$ é uma das iniciativas realizadas de maneira independente, sendo seus contribuidores, em sua maioria, especialistas em programação. A plataforma é anterior à pandemia, e tem como missão "tornar acessíveis os dados brasileiros de interesse público». Funciona como um repositório de dados públicos organizados e estruturados, de forma a facilitar a compreensão, a

13 Não está incluído o "Laboratório de Emergência - Covid-19».

14 Não está incluído o "Laboratório de Emergência - Covid-19».

15 https://brasil.io/Covid19/ 
apropriação e o uso pela sociedade. Na área dedicada aos dados da COVID-19, são exibidos os resultados da compilação diária dos boletins epidemiológicos das 27 Secretarias Estaduais de Saúde, tarefa realizada por uma força-tarefa formada por 40 pessoas, todas voluntárias.

Outro exemplo é a Rede Análise COVID1916, coletivo independente formado por mais de 170 pessoas, entre cientistas e profissionais de diferentes áreas (jornalismo, programação e ciência de dados, entre outras), que atuam voluntariamente. As ações do projeto incluem análise e modelagem de dados, coleta e análise de bancos de dados, e textos de divulgação científica, com foco na COVID-19. Algumas das análises publicadas no portal da Rede são resultado de trabalho colaborativo entre cientistas e não cientistas. Há ainda publicações cuja autoria não está informada, assinadas apenas com o nome da rede. O site traz também comentários sobre estudos em andamento, além de gráficos e outras formas de visualização de informação sobre os efeitos da pandemia no Brasil. Embora não seja uma plataforma aberta à contribuição de não integrantes, e não seja um projeto científico stricto sensu, há produção colaborativa de conhecimento, envolvendo múltiplos atores.

Abordagem distinta é a do Corona Cidades ${ }^{17}$, portal que oferece informações e consultoria gratuita à gestão pública de âmbito municipal na resposta à COVID-19. A iniciativa é mantida pelas organizações sem fins lucrativos Impulso ${ }^{18}$, Instituto de Estudos para Políticas de Saúde (IEPS) ${ }^{19}$ e Instituto Arapyaú ${ }^{20}$ e conta com a participação voluntária de profissionais com formações variadas.

Já o Painel Covid-19 nas Favelas ${ }^{21}$ foi criado pela ONG Voz das Comunidades ${ }^{22}$ para suprir a falta de informação sobre a evolução da epidemia nas favelas do município do Rio de Janeiro. Aqui, dados de di- ferentes fontes oficiais são desagregados e cruzados para identificação dos casos de contaminação e óbitos ocorridos especificamente nas favelas. O resultado é então divulgado no site da iniciativa de forma compreensível para o público em geral, com foco nos moradores das comunidades afetadas. Projeto com foco similar é o Favelas contra o Coronavírus - Simulador $^{23}$, ferramenta aberta desenvolvida por pesquisadores da Universidade Federal de São Carlos (UFSCar) em parceria com a iniciativa Favelas contra o Coronavírus ${ }^{24}$, formada por coletivos de comunicação comunitária, para possibilitar o entendimento pela população do impacto de políticas públicas de saúde (como disponibilidade hospitalar), no contexto da pandemia, em grupos sociais de baixa renda.

Foram identificadas também duas experiências baseadas no modelo de crowdsourcing. Uma delas é a iniciativa "Brasil sem Corona ${ }^{25}$, voltada para o mapeamento colaborativo de casos de COVID-19 e da evolução da doença no Brasil e desenvolvida pelas startups Colab ${ }^{26}$ e Epitrack ${ }^{27}$. Os dados que alimentam a plataforma são enviados pela população via um aplicativo para celular. 0 projeto está baseado na ideia de vigilância epidemiológica participativa, abordagem adotada por iniciativas similares ao redor do mundo que permite a construção de cenários epidemiológicos com participação cidadã.

A outra é a plataforma ColabCovid19 ${ }^{28}$, resultado de uma parceria entre a Universidade Federal do Paraná (UFPR) e a Universidade Federal do Amazonas (UFAM). A iniciativa se define como cartografia colaborativa e permite que a população informe, anonimamente, a existência de casos confirmados de COVID-19 e óbitos suspeitos pela doença na cidade em que moram. Seu objetivo é diagnosticar e combater as subnotificações de casos, para fins de pesquisa acadêmica. 0 projeto está hospedado na plataforma queniana Ushahidi ${ }^{29}$,

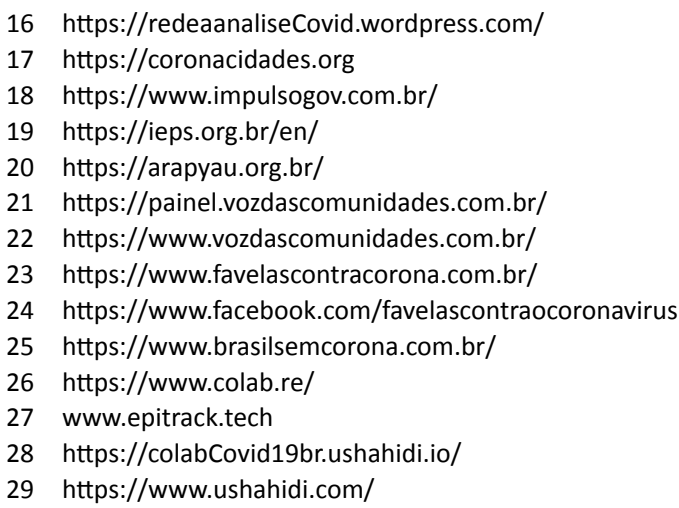


que possibilita a produção colaborativa de dados e informação por iniciativas e movimentos de organizações da sociedade civil.

\subsubsection{Fabricação cidadã}

O foco das iniciativas de fabricação cidadã para o enfrentamento da COVID-19 recaiu em desenvolvimento, produção e distribuição de respiradores, ventiladores e equipamentos de proteção individual (como máscaras e face shields ${ }^{30}$ ), geralmente de código aberto e baixo custo.

Uma característica dessas iniciativas é contar com parcerias e expertises diversas, particularmente a colaboração entre laboratórios universitários e espaços makers, muitos dos quais dispondo de impressoras 3D, facilitando o compartilhamento de infraestrutura e a troca de conhecimentos para o desenvolvimento e a produção de equipamentos crescentemente demandados por profissionais e serviços de saúde no contexto da pandemia. Um exemplo disso é a iniciativa Brasil contra o vírus $(B C V)^{31}$, rede de abrangência nacional formada por makers, fablabs, comunidades acadêmica e tecnológica, profissionais da saúde e representantes da indústria, resultado de uma articulação liderada por uma ex-aluna ${ }^{32}$ da Universidade Federal de São Paulo (Unifesp).

Frequentemente a participação de pessoas ligadas a instituições acadêmicas nas iniciativas de fabricação cidadã ocorre sem que exista vínculo oficial da iniciativa à instituição. Esse é caso da própria $B C V$, que recebeu apoio da Unifesp ${ }^{33}$, sem ser formalmente uma iniciativa desta universidade. Essa característica já havia sido apontada em estudo a respeito dos hackerspaces no Brasil (Martins e Albagli, 2020).

Trajetória similar à da BCV tem o Coletivo $\mathrm{Ar}$ Brasil $^{34}$, desdobramento do grupo de Facebook COVID-19 Air BRASIL - Fast production of assisted ventilation devices ${ }^{35}$, criado por um professor da Universidade Federal do Rio de Janeiro (UFRJ), em março de 2020, com o objetivo de reunir pessoas interessadas em desenvolver um respirador mecânico de código aberto e baixo custo, para uso pelo sistema de saúde brasileiro. O coletivo originado a partir do grupo no Facebook ${ }^{36}$ constituiu-se em oito núcleos estaduais (São Paulo, Minas Gerais, Rio de Janeiro, Rio Grande do Norte, Bahia, Paraná, Santa Catarina e Rio Grande do Sul) e um nacional ${ }^{37}$. Ainda que inspirado no projeto do professor da UFRJ e fundado por integrantes da rede iniciada por ele, o coletivo estabeleceu-se de modo independente àquela universidade.

Existem projetos com laços mais estreitos com a universidade, como o coletivo SOS 3D Covid19 ${ }^{38}$, baseado no Rio de Janeiro. Criado a partir da associação de pessoas com diferentes formações para produção e fornecimento de face shields a profissionais de saúde, o projeto teve apoio oficial da UFRJ e da Pontifícia Universidade Católica do Rio de Janeiro (PUC-Rio), a qual cedeu o espaço para a fabricação das viseiras. Já a iniciativa Cuid4r - Cuidar de quem cuida da gente ${ }^{39}$, cujo objetivo incluiu a produção de EPIs (face shield, óculos, máscaras de tecido) e de máscaras CPAP ${ }^{40}$, nasceu de uma ideia de uma professora do campus de Lagarto do Instituto Federal de Sergipe (IFS) ${ }^{41}$, tendo

30 Escudos faciais, espécie de viseira transparente produzida em acrílico para proteção do rosto. Apesar de existir expressão equivalente em português, o termo em inglês foi o que se popularizou entre as iniciativas, razão pela qual se optou por usá-lo neste artigo.

31 https://brcontraovirus.org/

32 https://g1.globo.com/bemestar/coronavirus/noticia/2020/03/20/brasileira-cria-rede-para-imprimir-em-3d-equipamentos-de-uti-usados-no-tratamento-contra-o-coronavirus.ghtml

33 https://www.unifesp.br/noticias-anteriores/item/4362-unifesp-apoia-projeto-de-rede-para-imprimir-em-3d-equipamentos-de-uti-usados-no-tratamento-contra-o-coronavirus

34 https://linktr.ee/Coletivoarbrasil

35 https://www.facebook.com/groups/235476464265909

36 https://www.facebook.com/coletivoarbrasil/photos/a.112878520354198/112879683687415

37 Dado de 05 de novembro de 2020.

38 https://www.sos3dCovid19.com.br/

39 https://www.cuid4r.com/

40 O CPAP é um aparelho compressor de ar usado no tratamento da apneia do sono, que se conecta ao indivíduo pelo uso de uma máscara ajustada ao nariz e à boca. O equipamento também é usado em ambiente hospitalar no auxílio a pacientes com dificuldade de respiração - e, no caso da covid-19, passou a ser adotado como alternativa à intubação. Diante da escassez das máscaras CPAP após o início da pandemia, surgiu a proposta de se adaptarem máscaras de mergulho para esse fim. (mais informações em http://portalarquivos2.saude. gov.br/images/pdf/2020/June/02/CabineProtecao-COVID19-atualizacao.pdf. Acesso em 08/11/2020)

41 Trata-se da profa. Stephanie Kamarry. 
recebido apoio do $\mathrm{IFS}^{42}$ e estabelecido parceria com a Universidade Federal de Sergipe (UFS) ${ }^{43}$.

O modelo usado para a fabricação das viseiras pela Cuid4r foi desenvolvido e registrado na Agência Nacional de Vigilância Sanitária (Anvisa) por outra iniciativa cidadã, a Motirõ ${ }^{44}$, em um exemplo de associações que se formam entre diferentes iniciativas. A Motirõ define-se como «um time de profissionais de diversas áreas, focados na construção de soluções técnicas de baixo custo e alto impacto e em oferecê-las gratuitamente para o tratamento da covid-19». Surgiu com o objetivo de adaptar máscaras de mergulho para equipamento de ventilação não invasiva - ideia que nasceu na Itália e inspirou alguns projetos semelhantes no Brasil - e depois acabou ampliando a atuação para a produção de EPIs. Trata-se de iniciativa totalmente independente, formada por profissionais da engenharia, do direito e da área da saúde, tendo polos nas cidades de Bauru, Botucatu, Campinas, Itapeva, São José dos Campos e São Paulo, todas no Estado de São Paulo.

Há casos ainda de iniciativas de startups da área médica, como o Breath4Life Project $t^{45}$, criado para o desenvolvimento de um ventilador mecânico para impressão $3 \mathrm{D}$ e usinagem, com o objetivo de suprir, de forma voluntária e sem fins lucrativos, a alta demanda por equipamentos hospitalares. O projeto é vinculado às empresas Anestech Innovation ${ }^{46}$ e Risinge HefestoMedtech, ambas integrantes da Eretz. bio $^{47}$, incubadora de projetos de inovação em saúde. Todos os desenhos de projeto, estudos, relatórios e manuais estão disponíveis para download no site da iniciativa. Outro exemplo é a Bioprint ${ }^{48}$, startup criada para o desenvolvimento de implantes ósseos por meio de impressão 3D, que direcionou seus equipamentos para a produção de face shields, estabelecendo parcerias com a comunidade open source e pesquisadores ${ }^{49}$.

A mobilização e a organização em rede de integrantes do movimento maker, apontada anteriormente, levou ao surgimento de outras iniciativas de fabricação, como o projeto "Cada Impresão Conta» ${ }^{50}$, para a produção de escudos faciais, a coalizão "Covida Maker ${ }^{51}$, para produção de tecnologias de saúde de código aberto, e até iniciativas individuais de espaços makers, como o «Me Viro» ${ }^{52}$, de Brasília (DF).

\subsubsection{Redes de solidariedade e apoio}

Em redes de solidariedade e apoio, foram encontrados grupos de Facebook, aplicativos e plataformas para conectar diferentes atores e fomentar o surgimento de redes de colaboração, captação de doações financeiras, atuação direta de apoio a grupos em situação de vulnerabilidade, oferta de hospedagem para profissionais de saúde. Em comum, as experiências nesta categoria valem-se do compartilhamento de informação para a construção de conhecimento que leva à ação.

A já mencionada conexão em rede identificada em algumas iniciativas de fabricação cidadã também é encontrada nas classificadas como redes de solidariedade e apoio. A maioria delas se formou de maneira independente, constituindo parcerias locais que contribuíssem para viabilização de suas ações, sem, no entanto, perderem seu caráter autônomo. Um caso de iniciativa independente formada em razão da COVID-19, organizada em rede e com parcerias locais é a UniãoBR, liga de núcleos estaduais independentes, também chamados União, que oferecem apoio à população em situação de vulnerabilidade e a profissionais da saúde. O objetivo da UniãoBR é dar apoio e orientação às demais Uniões, possibilitando a formação de novos núcleos. Em seu portal ${ }^{53}$, é possível encontrar a relação de todos os grupos estaduais e realizar doações financeiras.

Ao todo são 18 núcleos da UniãoBR, contabilizados cada qual como uma iniciativa individual para fins deste estudo ${ }^{54}$, localizados nos estados de Alagoas, Amazonas, Bahia, Ceará, Espírito Santo, Goiás, Maranhão,

42 https://opapajaca.com/2020/03/9689/

$43 \mathrm{http}: / /$ radioufs.ufs.br/conteudo/65051-coronavirus-ufs-produz-protetores-faciais-em-impressoras-3d-para-hospitais

44 http://www.motirosaude.com/

45 https://breath4lifeproject.com/

46 https://anestech.com.br/

47 https://eretz.bio/

48 http://www.bioprint.com.br/

49 https://www.instagram.com/p/B-lj-1mjlt7/

50 http://e-nablebrasil.org/wp/cadaimpressaoconta/

51 https://www.instagram.com/Covidamaker/

52 http://www.meviro.com.br/

53 https://www.movimentouniaobr.com.br/

54 Apesar de haver uma União nacional, a mesma não representa uma unificação de atuação de todas as uniões estaduais. O caráter autônomo dos núcleos torna cada um deles uma iniciativa própria, independente, resultando em objetivos, ações e parcerias diversas. 
Mato Grosso do Sul, Minas Gerais, Pará, Paraíba, Paraná, Pernambuco, Rio de Janeiro, Rio Grande do Norte, São Paulo (onde surgiu o primeiro núcleo) e Sergipe, e um atuante na região da Amazônia. Cada União age de maneira autônoma, estabelecendo parcerias com instituições locais para apoio às atividades, como a União PB, que atua ao lado do Ministério Público Federal, da Universidade Estadual da Paraíba e da Central Única de Favelas (CUFA) ${ }^{55}$, para identificação e atendimento de grupos sociais mais afetados pela pandemia. Quase todas são iniciativas independentes, exceto pela União Bahia, que é gerida pela Liga do Bem $^{56}$ e pelo Instituto ProBem ${ }^{57}$, motivo pelo qual foi considerada a única com vínculo institucional oficial.

Foram identificadas também plataformas independentes, criadas para dar visibilidade a iniciativas de enfrentamento da pandemia e para mobilizar grupos e pessoas desejosas de participar de tais ações. Uma delas é o Segura a Onda ${ }^{58}$, um guia online de iniciativas cidadãs e de inovação social de enfrentamento ao novo coronavírus, mantido por uma rede que se autodefine como «rede cidadã de apoio mútuo e auto-organização. A plataforma está estruturada na forma de um fórum de discussão organizado em temas, por meio do qual é possível a interação e o compartiIhamento de iniciativas voltadas ao enfrentamento da COVID-19. Trata-se da versão brasileira da experiência espanhola Frena la Curva ${ }^{59}$, que ganhou versões em diversos países da América Latina e da Europa.

Outra é a plataforma colaborativa Somos Muitxs ${ }^{60}$, que surgiu com a proposta de «visualizar a presença territorial das iniciativas de apoio às comunidades, periferias, favelas, pessoas em situação de vulnerabilidade e muitos outras, surgidas no cenário da pandemia de covid-19». A iniciativa traz em seu site a localização e visualização, no mapa do território brasileiro, das ações identificadas, bem como um formulário para cadastro de projetos.

55 https://www.cufa.org.br/

56 https://www.aligadobem.org/

57 https://www.instagram.com/probemsocialinvestment/

58 https://seguraaonda.com.br/

59 https://frenalacurva.net/

60 http://somosmuitxs.com/

61 https://www.facebook.com/groups/apandemiadobem/

62 https://www.facebook.com/groups/206461410581682/

63 https://www.institutomariellefranco.org/mapacoronanasperiferias

64 https://www.institutomariellefranco.org/

65 https://protegebr.org/

66 https://www.olabi.org.br/

67 https://www.engajar.redesolidaria.org.br/
Entre as demais iniciativas independentes, duas são grupos no Facebook que reúnem pessoas interessadas em participar de ações de solidariedade. O primeiro intitula-se $A$ pandemia do bem ${ }^{61}$ e está voltado para dar visibilidade a ações de solidariedade, como doações financeiras e de alimentos. O outro chama-se Apoio a autônomos - COVID1962 e foi criado com o objetivo de conectar comerciantes que perderam a renda devido à pandemia e pessoas dispostas a colaborar com recursos financeiros.

Nas iniciativas que possuem vínculos institucionais formais, nesta categoria, foram identificadas experiências ligadas a ONGs, startups, fablabs, instituições de pesquisa, associações de voluntariado e movimentos sociais e coletivos. Uma delas é o Mapa Corona nas Periferias $^{63}$, desenvolvido pelo Instituto Marielle Franco ${ }^{64}$ para dar visibilidade a iniciativas de combate ao coronavírus voltadas para favelas e periferias brasileiras. O portal traz um mapa do Brasil em que é possível visualizar as ações identificadas, além de um formulário para cadastro de novas ações. A Protege$B R^{65}$ é outra plataforma de mapeamento, para identificação e visibilização de ações de fabricação de EPIs, desenvolvida pelo fablab Olabi66. O site traz um mapa do território nacional com os projetos cadastrados e possibilita a conexão de instituições e profissionais de saúde com pessoas e grupos que tenham materiais e equipamentos para doar. Esse é um exemplo de iniciativa que está na interface entre duas categorias, fazendo referência também à fabricação.

Ainda no conjunto de experiências orientadas para formação de redes de solidariedade e apoio para o enfrentamento da pandemia está a Rede Solidária ${ }^{67}$, plataforma da Associação Brasileira de ONGs (Abong). Seu objetivo é conectar, por meio de seu portal, iniciativas existentes a pessoas que queiram se engajar em ações de enfrentamento da pandemia. Com uma ênfase similar, a plataforma de volun- 
tariado Atados valeu-se de sua experiência prévia de mobilização e de sua rede de apoio para estimular o desenvolvimento de ações de combate aos impactos da COVID-1968. Dentre as iniciativas associadas ao Atados, destacam-se a Tech4CovidBr ${ }^{69}$, movimento de voluntariado para o desenvolvimento de projetos de cunho tecnológico para o enfrentamento da COVID-19, e o projeto Rooms Against Covid Brasil ${ }^{70}$, plataforma por meio da qual pessoas podem oferecer acomodações temporárias a profissionais de saúde que não podem voltar para casa. Estas duas últimas são versões brasileiras de iniciativas de mesmo nome criadas em Portugal.

Por fim, cabe ressaltar uma iniciativa que não se enquadrou em nenhuma das três categorias estabelecidas pelo estudo, mas cujas características inovadoras merecem uma análise à parte, por sinalizar um outro conjunto de ações emergentes no campo da ciência em moldes de cocriação. Trata-se do Laboratório de Emergência - Covid-1971, criado pela Silo - Arte e Latitude Rural72, organização da sociedade civil que se dedica a acolher e a difundir projetos culturais em zonas rurais. O Laboratório constituiu-se em uma iniciativa temporária com vistas a fomentar o desenvolvimento de projetos participativos de inovação cidadã para o enfrentamento da pandemia, oriundos sobretudo das periferias rurais e urbanas, que adotassem licenças livres e pudessem ser replicados e adaptados a outros contextos. Para seleção dos projetos apoiados, foram lançadas três chamadas públicas ao longo do ano de 2020, todas elas divididas em duas etapas: chamada de projetos e chamada de colaboradores para os projetos selecionados. Cada convocatória estava direcionada a uma área temática diferente.

Dessa iniciativa surgiram outras 51 , compreendendo um amplo universo de abordagens e direcionamentos, incluindo projetos de dados e informações, de fabricação e de redes de solidariedade e apoio. Um exemplo de ação que nasceu dessa iniciativa é a Cartografia das Memórias ${ }^{73}$, plataforma cujo objetivo é registrar e preservar, através do recebimento de relatos orais, as memórias de vivências individuais e coletivas durante a pandemia. O resultado é um banco de memórias criado coletivamente, que aceita contribuições em português e outros idiomas. $\mathrm{O}$ site está disponível em português e inglês.

\section{CONCLUSÕES}

Iniciativas cidadãs de vários tipos têm despontado, no mundo inteiro, com o objetivo de ampliar a participação social no enfrentamento das questões postas pelo crescente número e amplitude de situações de emergência e desastre socioambientais e sanitárias. Tais ações baseiam-se, em grande parte, na produção colaborativa e no compartilhamento de dados e informações, mobilizando infraestruturas e ferramentas de informação e comunicação digitais, sobretudo plataformas e aplicativos web e de celular. Contribuem para o avanço e a disseminação de conhecimento, o engajamento social e as políticas públicas, relevantes à resposta e à prevenção das situações de crise.

Tais iniciativas têm sido analisadas como parte do escopo da ciência cidadã, a partir de uma linha interpretativa que adota uma definição mais ampla e flexível do conceito, para além da contribuição voluntária de não especialistas para projetos de pesquisa científica stricto sensu. Coloca-se assim a relevância de se repensar o arcabouço epistêmico da ciência cidadã (Hicks et ali., 2019), de modo a dar conta do alargamento de seu significado, bem como do reconhecimento de seu caráter situado e diverso, segundo o contexto em que se desenvolve e os objetivos a que se propõe.

Este estudo evidenciou uma diversidade de iniciativas de enfrentamento da pandemia da COVID-19, como uma situação de emergência sanitária, combinando metodologias de ciência cidadã com foco mais tradicional (em menor proporção) e formas de ação e participação cidadã típicas da mobilização voluntária em situações de emergência e desastre. De modo geral, elas orientaram-se não apenas para promover o engajamento cidadão nesse enfrentamento, como também para mobilizar uma inteligência coletiva no desenho de soluções.

A pesquisa revelou ainda uma variedade de atores que promovem essas iniciativas cidadãs, incluindo desde organizações mais estabelecidas, como insti-

68 https://www.atados.com.br/coronavirus

69 https://tech4Covid19.org.br/

70 https://www.roomsagainstCovid.com.br/

71 https://labdeemergencia.silo.org.br/

72 https://silo.org.br/

73 https://cartografiadasmemorias.org/ 
tuições acadêmicas, ONGs, e startups com interesses sociais, até grupos makers, organizações comunitárias e grupos de voluntariado sem vínculo ou estrutura institucional. Algumas dessas iniciativas requerem que seus promotores tenham conhecimento técnico, como é o caso das iniciativas de fabricação, ou ainda certas habilidades, como capacitação para raspagem, tratamento e análise de dados (este é o caso da Rede Análise Covid-19 e da Brasil.lo, entre outras).

Caberia, por outro lado, debruçar-se sobre as diferentes posições hierárquicas e bases epistêmicas nos sistemas de produção de conhecimento, que podem implicar relações desiguais ou dificuldades de colaboração entre os distintos partícipes dessas ações e suas redes. Para tanto, sugerem-se estudos de caso ou de caráter mais etnográfico, o que estava fora do escopo e dos objetivos desta pesquisa.

Observou-se também que parte das iniciativas teve um caráter temporário, tendo sido descontinuadas após um período de atuação, seja por falta de recursos, seja pela diminuição do engajamento e das contribuições voluntárias, ou ainda por se considerar que os propósitos iniciais haviam sido cumpridos. Essa característica é observada em iniciativas de ciência cidadã estabelecidas em outras situações de emergência e desastre, como também naquelas focadas em outras agendas (Albagli e Rocha, 2021).

Seria necessário estabelecer condições para que essas ações possam ser ampliadas e continuadas além do momento de eclosão da crise, evitando que se configurem como esforços provisórios e passageiros. Isso permitiria um melhor aproveitamento dos aprendizados e resultados alcançados, tanto nos desdobramen- tos posteriores aos momentos de resposta à emergência e a suas implicações de mais longo alcance, como no enfrentamento de eventos dessa natureza em outras questões. Por exemplo, os ensinamentos obtidos em iniciativas de ciência cidadã e afins relacionadas a enfermidades como aids, ebola e zika vírus teriam sido mais bem conhecidos e replicados caso houvesse condições para sua continuidade ou desdobramentos posteriores. Este é o caso também de outras vertentes da ciência aberta.

Por fim, a existência de políticas públicas de apoio à ciência cidadã, dessa perspectiva ampla, pode desempenhar papel crucial, particularmente na provisão de infraestruturas que deem suporte à sua realização de modo autônomo e contínuo.

\section{AGRADECIMENTOS}

A pesquisa que resultou neste artigo contou com o apoio do Instituto Brasileiro de Informação em Ciência e Tecnologia (IBICT) e do Conselho Nacional de Desenvolvimento Científico e Tecnológico (CNPq). Agradecimentos também a/os pareceristas da primeira versão deste artigo.

\section{DISPONIBILIDADE DE DADOS DEPOSITADOS}

Planilha com as informações das iniciativas cidadãs brasileiras analisadas no estudo, incluindo nome, URL, foco, vínculo institucional, ano de criação e país. Depositado em: Zenodo, https://zenodo.org. Código identificador: 10.5281/zenodo.4277899. Licença de uso e distribuição: Creative Commons Attribution 4.0 International License

\section{BIBLIOGRAFIA}

Albagli, Sarita (2015). Ciência aberta em questão. In: Albagli, Sarita, Maciel, Maria Lucia Abdo, Alexandre Hannud. (orgs.). Ciência aberta, questões abertas. Brasília: Ibict; Rio de Janeiro: Unirio, pp 9-25. Disponível em: http:// livroaberto.ibict.br/handle/1/1060

Albagli, Sarita e Rocha, Luana (2021). Ciência cidadã no Brasil: um estudo exploratório. In: Borges, Maria Manuel e Casado, Elías Sanz. (orgs.). Sob a lente da Ciência aberta: olhares de Portugal, Espanha e Brasil. Coimbra: Universidade de Coimbra, pp. 489-511.

Albagli, Sarita e Iwama, Allan Yu [no prelo]. Citizen science, the right to research and climate change challenges: a study in the Southern coast of Brazil. Humanities and Social Sciences Communications.

Abellán-López, María Ángeles (2021). El cambio climático: negacionismo, escepticismo y desinformación. Tabula rasa, (37): 283-301. https://doi. org/10.25058/20112742.n37.13

Bezerra, Arthur Coelho; Schneider, Marco e Saldanha, Gustavo Silva (2019). Competência crítica em informação como crítica à competência em informação. Informação \& Sociedade 29 (3). https://periodicos.ufpb.br/ojs2/index. php/ies/article/view/45026

Black, Peter e Butler, Colin D. (2014). One health in a world with climate change.
Rev. sci. tech. Off. int. Epiz., 33 (2): 465473. doi: 10.20506/rst.33.2.2293.

Bowser, Anne; Parker, Alison e Long, Alex. Citizen Science and COVID-19: The Power of the (Distanced) Crowd. Ctrl Forward: blog of the Science and Technology Innovation Program at the Wilson Center. Disponível em: https:// www.wilsoncenter.org/blog-post/ citizen-science-and-covid-19-powerdistanced-crowd [data de publicação: 22 junho 2020; data de consulta: 17 dezembro 2020].

Bonney, Rick; Cooper, Caren; Dickinson, Janis; Kelling, Steve; Phillips, Tina; Rosenberg, Keneth V. e Shirk, Jennifer (2009). Citizen science: a developing tool for expanding science knowledge and scientific 
literacy. Bioscience, 59 (11): 977-984. https://doi.org/10.1525/bio.2009.59.11.9

Calyx, Cobi (2020). Sustaining Citizen Science beyond an Emergency. Sustainability, 12 (11): 4522. doi:10.3390/ su12114522

Chan, Leslie; Okune, Angela; Hillyer, Becky; Albornoz, Denise e Posada, Alejandro. (eds.) (2019). Contextualizing openness: situating open science. Ottawa: University of Ottawa Pres.

Clinio, Anne; Martins, Beatriz; Metzker Netto, Christiana; Tavares, Luiz Eduardo; Oliveira, Maria de Lourdes Silva de e Guimarães, Ricardo Marques da Silva Martins (2020). Segura a Onda: experiência brasileira de mapeamento de iniciativas cidadãs no enfrentamento da pandemia da Covid-19. Liinc em Revista, 16 (2). https://doi.org/10.18617/ liinc.v16i2.5386

Conrad, Cathy C.; Hilchey, Krista G. (2010). A review of citizen science and community-based environmental monitoring: issues and opportunities. Environmental Monitoring and Assessment, 176 (14): 273-291. https://doi.org/10.1007/ s10661-010-1582-5

Dickinson, Janis L.; Shirk, Jennifer; Bonter, David; Bonney, Rick; Crain, Rhiannon L.; Martin, Jason; Phillips, Tina e Purcell, Karen (2012). The current state of citizen science as a tool for ecological research and public engagement. Frontiers in Ecology and the Environment, 10 (6): 291297. https://doi.org/10.1890/110236

Flores, Reymund e Asuncion, Xavier Venn (2020). Toward an improved risk/ crisis communication in this time of COVID-19 pandemic: a baseline study for Philippine local government units. JCOM, 19 (07): A09. https://doi. org/10.22323/2.19070209.

Hacklay, Muki (2015). Citizen Science and Policy: an European perspective. Washington, DC: Woodrow Wilson International Center for Scholars.

Hicks, Anna; Barclay, Jenni; Chilvers, Jason; Armijos, M. Teresa; Oven, Katie; Simmons, Peter e Haklay, Muki (2019). Global mapping of Citizen science projects for disaster risk reduction. Front. Earth
Sci. 7:226. https://doi.org/10.3389/ feart.2019.00226

Horton, Richard (2020). Offline: COVID-19 is not a pandemic. Lancet, $396: 874$

Hussain, Azhar; Ali, Syed; Ahmed, Madiha e Hussain, Sheharyar (2018). The Anti-vaccination Movement: A Regression in Modern Medicine. Cureus, 10(7), e2919. https://doi.org/10.7759/cureus.2919

Irwin, Alan (1995). Citizen science: a study of people, expertise and sustainable development. Londres \& Nova York: Routledge.

Lafuente, Antonio e Estalella, Adolfo (2015). Modos de ciencia: pública, abierta y común. In: Albagli, Sarita; Maciel, Maria Lucia e Abdo, Alexandre Hannud. (orgs.). Ciência aberta, questões abertas. Brasília: IBICT; Rio de Janeiro: Unirio. pp. 27-58. Disponível em: http:// livroaberto.ibict.br/handle/1/1060

Lafuente, Antonio (2018). Laboratorios ciudadanos y nueva institucionalidad. Agenda Cultural Alma Máter, (256): 1920. Disponível em: https://revistas.udea. edu.co/index.php/almamater/article/ view/334573

Lasser, Jana; Ahne, Verena; Heiler, Georg; Klimek, Peter; Metzler, Hannah; Reisch, Tobias; Sprenger, Martin; Thurner, Stegan e Sorger, Johannes (2020). Complexity, transparency and time pressure: practical insights into science communication in times of crisis. JCOM 19 (05): N01. https://doi.org/10.22323/2.19050801

Marchezini, Victor; Horita, Flavio Eduardo Aoki; Matsuo, Patricia Mie; Trajber, Raquel; Trejo-Rangel, Miguel Angel e Olivato, Debora (2018). A Review of Studies on Participatory Early Warning Systems (P-EWS): Pathways to Support Citizen Science Initiatives. Front. Earth Sci. 6:184. https://doi. org/10.3389/feart.2018.00184

Martins, Beatriz Cintra (2017). Hackerspaces, ciência cidadã e ciência comum: apontamentos para uma articulação. Liinc em Revista, 13 (1): 59-71. https:// doi.org/10.18617/liinc.v13i1.3752

Martins, Beatriz Cintra e Albagli, Sarita (2020). Hackerspace movement: a study of the Brazilian experience. Em Questão, (26) 1: 161-185. http://dx.doi. org/10.19132/1808-5245261.161-185
Metcalfe, Jenni; Riedlinger, Michelle; Bauer, Martin W.; Chakraborty, Anwesha; Gascoigne, Toss; Guenther, Lars; Joubert, Marina; Kaseje, Margaret; Herrera-Lima, Susana; Revuelta, Gema; Riise, Jan e Schiele, Bernard (2020). The COVID-19 mirror: reflecting science-society relationships across 11 countries. JCOM, 19 (07): A05. https:// doi.org/10.22323/2.19070205.

Oliveira, Thaiane Moreira de (2020). Como enfrentar a desinformação científica? Desafios sociais, políticos e jurídicos intensificados no contexto da pandemia. Liinc em Revista, Rio de Janeiro, 16 (2): e5374. https://doi.org/10.18617/liinc. v16i2.5374

Rios, Rafael S.; Zheng, Kenneth I. e Zheng, Ming-Hua (2020). Data sharing during COVID-19 pandemic: what to take away. Expert Review of Gastroenterology \& Hepatology, 14 (12):1125-1130. https://doi.org/10.1080/17474124.20 20.1815533

Rodrigues, Karina Furtado; Carpes, Mariana Montez e Raffagnato, Carolina Gomes (2020). Preparação e resposta a desastres do Brasil na pandemia da COVID-19. Rev. Adm. Pública, 54 (4): 614634. http://dx.doi.org/10.1590/0034761220200291

Smith, Adrian; Fressoli, Mariano; Abrol, Dinesh; Arond, Elisa e Ely, Adrian (2017). Grassroots Innovation Movements: Pathways to Sustainability. Londres: Routledge.

Stevens, Matthias; Vitos, Michalis; Altenbuchner, Julia; Conquest, Gillian; Lewis, Jerome e Haklay, Muki (2014). Taking participatory Citizen science to extremes. IEE Pervasive Computing, 13(2): 20-29. https://doi.org/10.1109/MPRV.2014.37

Tse, Edwin G.; Klug, Dana M. e Todd, Matthew H. (2020). Open science approaches to COVID-19 [version 1; peer review: 2 approved]. F1000Research, 9:1043 https://doi.org/10.12688/f1000research.26084.1

Velho, Lea (2011). Conceitos de ciência e a política científica, tecnológica e de inovação. Sociologias, 13 (26): 128153. https://doi.org/10.1590/S151745222011000100006 